\title{
Analysis of Subgradient Extragradient Iterative Schemes for Variational Inequalities
}

\author{
Danfeng Wu, ${ }^{1}$ Li-Jun Zhu $\mathbb{D},{ }^{2}$ Zhuang Shan $\mathbb{D}^{1},{ }^{1}$ and Tzu-Chien Yin $\mathbb{D}^{3}$ \\ ${ }^{1}$ School of Mathematics and Information Science, North Minzu University, Yinchuan 750021, China \\ ${ }^{2}$ The Key Laboratory of Intelligent Information and Big Data Processing of NingXia, North Minzu University, \\ Yinchuan 750021, China \\ ${ }^{3}$ Research Center for Interneural Computing, China Medical University Hospital, China Medical University, \\ Taichung 40402, Taiwan
}

Correspondence should be addressed to Li-Jun Zhu; zljmath@outlook.com and Tzu-Chien Yin; yintzuchien@mail.cmuh.org.tw Received 9 October 2021; Accepted 20 October 2021; Published 5 November 2021

Academic Editor: Liya Liu

Copyright (c) 2021 Danfeng Wu et al. This is an open access article distributed under the Creative Commons Attribution License, which permits unrestricted use, distribution, and reproduction in any medium, provided the original work is properly cited.

In this paper, we investigate the monotone variational inequality in Hilbert spaces. Based on Censor's subgradient extragradient method, we propose two modified subgradient extragradient algorithms with self-adaptive and inertial techniques for finding the solution of the monotone variational inequality in real Hilbert spaces. Strong convergence analysis of the proposed algorithms have been obtained under some mild conditions.

\section{Introduction}

Let $\mathrm{H}$ be a real Hilbert space and $\mathrm{S} \in \mathrm{H}$ be a nonempty closed convex subset. Let $f: \mathrm{H} \longrightarrow \mathrm{H}$ be an operator. In this work, we investigate the following variational inequality problem (VIPs):

$$
\text { find a point } u^{\ddagger} \in \mathrm{S}, \quad \text { s.t. }\left\langle f\left(u^{\ddagger}\right), x-u^{\ddagger}\right\rangle \geq 0, \forall x \in \mathrm{S} \text {. }
$$

Denote by Sol $(\mathrm{S}, f)$ the solution set of (1). The VIPs is an important tool to study various problems in the domain of mechanics, optimization, transportation, fixed point, economics equilibrium, contract problems in elasticity, and other branches of mathematics, see [1-17]. Therefore, VIPs have received much attention by many scholars, see [18-30]. There are a variety of methods to solve the VIPs, such as regularization method and projection method [31-39]. In this work, we focus on projection method.

As we all know that the gradient projection algorithm is the simplest and oldest method $([40,41])$, the method is defined as follows:

$$
u^{k+1}=P_{S}\left(u^{k}-\gamma f\left(u^{k}\right)\right)
$$

where $P_{\mathcal{S}}: \mathrm{H} \longrightarrow \mathrm{S}$ is the metric projection and $\gamma$ is some positive number.

In order to obtain a convergent result, this algorithm requires that the operator $f$ is strongly monotone. In order to avoid the strong monotonicity hypothesis, Korpelevich [42] proposed the extragradient algorithm which is stated as follows:

$$
\left\{\begin{array}{l}
x^{k}=P_{\mathrm{S}}\left(u^{k}-\gamma f\left(u^{k}\right)\right), \\
u^{k+1}=P_{\mathrm{S}}\left(u^{k}-\gamma f\left(x^{k}\right)\right),
\end{array}\right.
$$

where $\gamma \in(0,(1 / L))$ and operator $f$ is monotone and Lipschitz continuous in $\mathrm{H}$.

Note that the algorithm (3) calculates two projections on $\mathrm{S}$ in each iteration. If the set $\mathrm{S}$ is more complicated, there will be a lot of calculations. In order to overcome this difficulty, Censor et al. [43] constructed a half space with subdifferentiation and proposed subgradient extragradient method which is defined by 


$$
\left\{\begin{array}{l}
x^{k}=P_{S}\left(u^{k}-\gamma f\left(u^{k}\right)\right), \\
T^{k}=\left\{z \in \mathrm{H} \mid\left\langle u^{k}-\gamma f\left(u^{k}\right)-x^{k}, z-x^{k}\right\rangle \leq 0\right\} \\
u^{k+1}=P_{T^{k}}\left(u^{k}-\gamma f\left(x^{k}\right)\right) .
\end{array}\right.
$$

Recently, Dong et al. [44] improved the algorithm (4) with self-adaptive stepsize which generates a sequence $\left\{u^{k}\right\}$ by the following form:

$$
\left\{\begin{array}{l}
x^{k}=P_{\delta}\left(u^{k}-\gamma^{k} f\left(u^{k}\right)\right), \\
\gamma^{k}\left\|f\left(u^{k}\right)-f\left(x^{k}\right)\right\| \leq \sigma\left\|u^{k}-x^{k}\right\|, \quad \forall \sigma \in(0,1), \\
T^{k}=\left\{z \in \mathrm{H}\left\langle u^{k}-\gamma^{k} f\left(u^{k}\right)-x^{k}, z-x^{k}\right\rangle \leq 0\right\}, \\
u^{k+1}=P_{T^{k}}\left(u^{k}-\tau \zeta^{k} \gamma^{k} f\left(x^{k}\right)\right),
\end{array}\right.
$$

where $\zeta^{k}=\left(\left\langle u^{k}-x^{k}, \kappa\left(u^{k}, x^{k}\right)\right\rangle /\left\|\kappa\left(u^{k}, x^{k}\right)\right\|^{2}\right)$ and $\kappa\left(u^{k}\right.$, $\left.x^{k}\right)=\left(u^{k}-x^{k}\right)-\gamma^{k}\left(f\left(u^{k}\right)-f\left(x^{k}\right)\right)$.

Weak convergence of Algorithm (5) has been obtained. Motivated and inspired by the above work, in this paper, we continue to investigate iterative algorithms for solving the monotone variational inequality in Hilbert spaces. We construct two modified subgradient extragradient algorithms for finding the solution of the monotone variational inequality. Our algorithms combine self-adaptive technique and inertial method. Under some mild conditions, we prove that the proposed algorithms converge strongly to a solution of the monotone variational inequality.

The organizational structure of this paper is as follows. In Section 2, we present some definitions and preliminary results, which will be used in further analysis of the proposed algorithms. In Section 3, we proposed two modified subgradient extragradient algorithms and prove strong convergence theorems.

\section{Preliminaries}

Let $C$ be a nonempty closed convex subset of a real Hilbert space $H$. Use " $\rightarrow$ " and “ $\longrightarrow$ " to denote weak and strong convergence, respectively. Let $\left\{x^{k}\right\}$ be a sequence in $H$. We use $\omega_{w}\left(x^{k}\right)$ to denote the set of all weak cluster points of $\left\{x^{k}\right\}$, i.e.,

$\omega_{w}\left(x^{k}\right)=\left\{x^{\dagger}: \exists\left\{x^{k_{i}}\right\} \subset\left\{x^{k}\right\}\right.$ such that $x^{k_{i}} \rightarrow x^{\dagger}$ as $\left.i \longrightarrow \infty\right\}$.

For $\forall u, v \in H$, and $\lambda \in \mathrm{R}$, the following results hold

$$
\begin{aligned}
\|u+v\|^{2} \leq & \|u\|^{2}+2\langle v, u+v\rangle, \\
\|\lambda u+(1-\lambda) v\|^{2}= & \lambda\|u\|^{2}+(1-\lambda)\|v\|^{2} \\
& -\lambda(1-\lambda)\|u-v\|^{2} .
\end{aligned}
$$

Definition 1. Let $f: H \longrightarrow H$ be an operator. Recall that the operator $f$ is said to be

(i) Monotone if

$$
\langle f(u)-f(v), u-v\rangle \geq 0, \quad \forall u, v \in H .
$$

(ii) Strongly monotone if there exists $\gamma>0$ s.t.

$$
\langle f(u)-f(v), u-v\rangle \geq \gamma\|u-v\|^{2}, \quad \forall u, v \in H .
$$

(iii) $L$-Lipschitz continuous if there exists $L>0$ s.t.

$$
\|f(u)-f(v)\| \leq L\|u-v\|, \quad \forall u, v \in H .
$$

If $L<1, f$ is said to be $L$-contractive.

Let $C$ be a nonempty closed convex subset of a real Hilbert space $H$. For any $x \in H$, there exists a unique point $P_{C}(x) \in C$ such that

$$
\left\|x-P_{C}(x)\right\| \leq\|y-x\|, \quad \forall y \in C .
$$

It is well known that $P_{C}$ satisfies [45]

$$
\begin{gathered}
\left\langle x-P_{C}(x), y-P_{C}(x)\right\rangle \leq 0, \\
\left\|x-P_{C}(x)\right\|^{2}+\left\|y-P_{C}(x)\right\|^{2} \leq\|x-y\|^{2},
\end{gathered}
$$

for all $x \in H$ and $y \in C$.

Lemma 1 (see [46]). Let $\left\{b^{k}\right\}$ be a real number sequence. Suppose that there exists a subsequence $\left\{b^{k_{m}}\right\}$ of $\left\{b^{k}\right\}$ such that $b^{k_{m}}<b^{k_{m}+1}$ for all $m \in \mathbb{N}$. Define the sequence $\{\gamma(k)\}$ as follows:

$$
\gamma(k)=\max \left\{n \in \mathbb{N} \mid \bar{k}_{0} \leq n \leq k, b^{n} \leq b^{n+1}\right\},
$$

for each $k \geq \bar{k}_{0}$. Then, the following inequality holds:

$$
0 \leq b^{k} \leq b^{\gamma(k)+1}
$$

for each $k \geq \bar{k}_{0}$. Further, for all $k \geq \bar{k}_{0}$, the sequence $\{\gamma(k)\}$ is nondecreasing and

$$
\lim _{k \longrightarrow \infty} \gamma(k)=+\infty
$$

Lemma 2 (see [33]). Suppose that the sequence $\left\{\delta^{k}\right\}$ of real numbers is nonnegative and there exists $k_{0} \in \mathbb{N}$ such that

$$
\delta^{k+1} \leq\left(1-\gamma^{k}\right) \delta^{k}+\gamma^{k} l^{k}
$$

for each $k \geq k_{0}$, where the sequences $\left\{\gamma^{k}\right\}$ and $\left\{l^{k}\right\}$ satisfy the following conditions:

$$
\begin{aligned}
\left\{\gamma^{k}\right\} & \subset(0,1), \\
\lim _{k \longrightarrow \infty} \gamma^{k} & =0, \\
\sum_{k=1}^{\infty} \gamma^{k} & =\infty, \\
\limsup _{k \rightarrow \infty} l^{k} & \leq 0 .
\end{aligned}
$$

Then, $\lim _{k \longrightarrow \infty} \delta^{k}=0$. 


\section{Main Result}

In this section, we present our main results.

Let $S$ be a nonempty closed convex subset of a real Hilbert space $H$. Suppose that the following three conditions are satisfied:

$(\mathrm{C} 1)$ : the set $\operatorname{Sol}(\mathrm{S}, f)$ is not empty;

(C2): the operate $f$ is monotone;

(C3): the operate $f$ is $L$-Lipschitz continuous.

Let $\sigma, \rho \in(0,1), \tau \in(0,2)$, and $\gamma^{0}>0$ be four constants. Let $\left\{\theta^{k}\right\},\left\{\varepsilon^{k}\right\} \subset(0,1)$, and $\left\{\lambda^{k}\right\} \subset[a, b] \subset(0,1)$ be three sequences, satisfying

$$
\begin{aligned}
\sum_{n=1}^{\infty} \theta^{k} & =+\infty, \\
\lim _{k \longrightarrow \infty} \theta^{k} & =0, \\
\varepsilon^{k} & =o\left(\theta^{k}\right) .
\end{aligned}
$$

Next, we introduce an iterative algorithm for solving (1).

Lemma 3. If $x^{k}=v^{k}$ or $\kappa\left(v^{k}, x^{k}\right)=0$ in Algorithm 1, then $x^{k} \in \operatorname{Sol}(\mathrm{S}, f)$.

Proof. Since $f$ is $L$-Lipschitz continuous, we obtain

$$
\begin{aligned}
\left\|\kappa\left(v^{k}, x^{k}\right)\right\| & =\left\|v^{k}-x^{k}-\gamma^{k}\left(f\left(v^{k}\right)-f\left(x^{k}\right)\right)\right\| \\
& \geq\left\|v^{k}-x^{k}\right\|-\gamma^{k}\left\|f\left(v^{k}\right)-f\left(x^{k}\right)\right\| \\
& \geq\left\|v^{k}-x^{k}\right\|-\gamma^{k} L\left\|v^{k}-x^{k}\right\|=\left(1-\gamma^{k} L\right)\left\|v^{k}-x^{k}\right\|, \\
\left\|\kappa\left(v^{k}, x^{k}\right)\right\| & =\left\|v^{k}-x^{k}-\gamma^{k}\left(f\left(v^{k}\right)-f\left(x^{k}\right)\right)\right\| \\
& \leq\left\|v^{k}-x^{k}\right\|+\gamma^{k}\left\|f\left(v^{k}\right)-f\left(x^{k}\right)\right\| \\
& \leq\left\|v^{k}-x^{k}\right\|+\gamma^{k} L\left\|v^{k}-x^{k}\right\|=\left(1+\gamma^{k} L\right)\left\|v^{k}-x^{k}\right\| .
\end{aligned}
$$

It follows that

$$
\left(1-\gamma^{k} L\right)\left\|v^{k}-x^{k}\right\| \leq\left\|\kappa\left(v^{k}, x^{k}\right)\right\| \leq\left(1+\gamma^{k} L\right)\left\|v^{k}-x^{k}\right\| .
$$

Consequently, $v^{k}=x^{k} \Leftrightarrow \kappa\left(v^{k}, x^{k}\right)=0$. Furthermore, if $v^{k}=x^{k}$ or $\kappa\left(v^{k}, x^{k}\right)=0$, we have

$$
x^{k}=P_{\mathrm{S}}\left(x^{k}-\gamma^{k} f\left(x^{k}\right)\right) .
$$

Combining (13) and (23), we get

$$
\left\langle x^{k}-\gamma^{k} f\left(x^{k}\right)-x^{k}, x^{k}-z\right\rangle \geq 0, \quad \forall z \in \mathrm{S},
$$

which implies that

$$
\left\langle f\left(x^{k}\right), z-x^{k}\right\rangle \geq 0, \quad \forall z \in \mathrm{S} .
$$

This completes the proof.
Lemma 4. The sequence $\left\{\gamma^{k}\right\}_{k=0}^{\infty}$ generated by Algorithm 1 is monotonically decreasing, and $\gamma^{k} \leq \min \left\{\gamma^{0},(\sigma / L)\right\}$ for each $k \geq 0$.

Proof. Obviously, by the definition of $\left\{\gamma^{k+1}\right\}$, we have $\left\{\gamma^{k}\right\}$ is a monotonically decreasing sequence. Then, $\gamma^{k} \geq \gamma^{0}, \forall n>0$. Since $f$ is Lipschitz continuous, we have

$$
\left\|f\left(u^{k}\right)-f\left(x^{k}\right)\right\| \leq L\left\|u^{k}-x^{k}\right\| .
$$

In the case of $f\left(u^{k}\right) \neq f\left(x^{k}\right)$, we have

$$
\frac{\sigma\left\|u^{k}-x^{k}\right\|}{\left\|f\left(u^{k}\right)-f\left(x^{k}\right)\right\|} \geq \frac{\sigma}{L} \text {. }
$$

Obviously, the lower bound of $\left\{\gamma^{k}\right\}$ is $\min \left\{\gamma^{0},(\sigma / L)\right\}$. This completes the proof.

Lemma 5. Let $\left\{\zeta^{k}\right\}$ be the sequence generated by Algorithm 1 . Then, we have

$$
\zeta^{k} \geq \frac{1-\sigma}{1+\sigma^{2}}
$$

Proof. Combining Lemma 4 and Cauchy-Schwartz inequality, we have

$$
\begin{aligned}
\left\langle v^{k}-x^{k}, \kappa\left(v^{k}, x^{k}\right)\right\rangle & =\left\langle v^{k}-x^{k}, v^{k}-x^{k}-\gamma^{k}\left(f\left(v^{k}\right)-f\left(x^{k}\right)\right)\right\rangle \\
& =\left\|v^{k}-x^{k}\right\|^{2}-\gamma^{k}\left\langle v^{k}-x^{k}, f\left(v^{k}\right)-f\left(x^{k}\right)\right\rangle \\
& \geq\left\|v^{k}-x^{k}\right\|^{2}-\gamma^{k}\left\|v^{k}-x^{k}\right\|\left\|f\left(v^{k}\right)-f\left(x^{k}\right)\right\| \\
& \geq\left\|v^{k}-x^{k}\right\|^{2}-\gamma^{k} L\left\|v^{k}-x^{k}\right\|^{2} \\
& =\left(1-\gamma^{k} L\right)\left\|v^{k}-x^{k}\right\|^{2} \\
& \geq(1-\sigma)\left\|v^{k}-x^{k}\right\|^{2} .
\end{aligned}
$$

Since $f$ is monotone and Lipschitz continuous, then we obtain

$$
\begin{aligned}
\left\|\kappa\left(v^{k}, x^{k}\right)\right\|^{2}= & \left\|v^{k}-x^{k}-\gamma^{k}\left(f\left(v^{k}\right)-f\left(x^{k}\right)\right)\right\|^{2} \\
= & \left\|v^{k}-x^{k}\right\|^{2}+\left(\gamma^{k}\right)^{2}\left\|f\left(v^{k}\right)-f\left(x^{k}\right)\right\|^{2} \\
& -2 \gamma^{k}\left\langle v^{k}-x^{k}, f\left(v^{k}\right)-f\left(x^{k}\right)\right\rangle \\
\leq & \left\|v^{k}-x^{k}\right\|^{2}+\left(\gamma^{k}\right)^{2} L^{2}\left\|v^{k}-x^{k}\right\|^{2} \\
= & \left(1+\left(\gamma^{k}\right)^{2} L^{2}\right)\left\|v^{k}-x^{k}\right\|^{2} \\
\leq & \left(1+\sigma^{2}\right)\left\|v^{k}-x^{k}\right\|^{2} .
\end{aligned}
$$

From (29) and (30), we have

$$
\zeta^{k}=\frac{\left\langle v^{k}-x^{k}, \kappa\left(v^{k}, x^{k}\right)\right\rangle}{\left\|\kappa\left(v^{k}, x^{k}\right)\right\|^{2}} \geq \frac{1-\sigma}{1+\sigma^{2}} .
$$


This completes the proof.

Lemma 6. Let $u^{\ddagger} \in \operatorname{Sol}(S, f)$. Then,

$$
\begin{gathered}
\left\|t^{k}-u^{\ddagger}\right\|^{2} \leq\left\|v^{k}-u^{\ddagger}\right\|^{2}-\left\|\left(v^{k}-t^{k}\right)-\tau \zeta^{k} \kappa\left(v^{k}, x^{k}\right)\right\|^{2} \\
-\tau(2-\tau)\left(\zeta^{k}\right)^{2}\left\|\kappa\left(v^{k}, x^{k}\right)\right\|^{2} .
\end{gathered}
$$

Proof. From (13) and Algorithm 1, we have

$$
\begin{aligned}
\left\|t^{k}-u^{\ddagger}\right\|^{2} \leq & \left\|P_{T^{k}}\left(v^{k}-\tau \zeta^{k} \gamma^{k} f\left(x^{k}\right)\right)-P_{T^{k}} u^{\ddagger}\right\|^{2} \\
\leq & \left\langle t^{k}-u^{\ddagger}, v^{k}-\tau \zeta^{k} \gamma^{k} f\left(x^{k}\right)-u^{\ddagger}\right\rangle \\
= & \frac{1}{2}\left\|t^{k}-u^{\ddagger}\right\|^{2}+\frac{1}{2}\left\|v^{k}-\tau \zeta^{k} \gamma^{k} f\left(x^{k}\right)-u^{\ddagger}\right\|^{2} \\
& -\frac{1}{2}\left\|t^{k}-v^{k}+\tau \zeta^{k} \gamma^{k} f\left(x^{k}\right)\right\|^{2} \\
= & \frac{1}{2}\left\|t^{k}-u^{\ddagger}\right\|^{2}+\frac{1}{2}\left\|v^{k}-u^{\ddagger}\right\|^{2} \\
& +\frac{1}{2} \tau^{2}\left(\zeta^{k}\right)^{2}\left(\gamma^{k}\right)^{2}\left\|f\left(x^{k}\right)\right\|^{2} \\
& -\left\langle v^{k}-u^{\ddagger}, \tau \zeta^{k} \gamma^{k} f\left(x^{k}\right)\right\rangle-\frac{1}{2}\left\|t^{k}-v^{k}\right\|^{2} \\
& -\frac{1}{2} \tau^{2}\left(\zeta^{k}\right)^{2}\left(\gamma^{k}\right)^{2}\left\|f\left(x^{k}\right)\right\|^{2}-\left\langle t^{k}-v^{k}, \tau \zeta^{k} \gamma^{k} f\left(x^{k}\right)\right\rangle \\
= & \frac{1}{2}\left\|t^{k}-u^{\ddagger}\right\|^{2}+\frac{1}{2}\left\|v^{k}-u^{\ddagger}\right\|^{2}-\frac{1}{2}\left\|t^{k}-v^{k}\right\|^{2} \\
& -\left\langle t^{k}-u^{\ddagger}, \tau \zeta^{k} \gamma^{k} f\left(x^{k}\right)\right\rangle . \\
&
\end{aligned}
$$

It follows that

$$
\begin{gathered}
2\left\|t^{k}-u^{\ddagger}\right\|^{2} \leq\left\|t^{k}-u^{\ddagger}\right\|^{2}+\left\|v^{k}-u^{\ddagger}\right\|^{2}-\left\|t^{k}-v^{k}\right\|^{2} \\
-2 \tau \zeta^{k} \gamma^{k}\left\langle t^{k}-u^{\ddagger}, f\left(x^{k}\right)\right\rangle,
\end{gathered}
$$

or equivalently

$$
\left\|t^{k}-u^{\ddagger}\right\|^{2} \leq\left\|v^{k}-u^{\ddagger}\right\|^{2}-\left\|t^{k}-v^{k}\right\|^{2}-2 \tau \zeta^{k} \gamma^{k}\left\langle t^{k}-u^{\ddagger}, f\left(x^{k}\right)\right\rangle .
$$

We deduce from $x^{k} \in C$ and $u^{\ddagger} \in \operatorname{Sol}(S, f)$ that $\left\langle f\left(u^{\ddagger}\right), x^{k}-u^{\ddagger}\right\rangle \geq 0$. It follows from the monotonicity of operator $f$ that $\left\langle f\left(x^{k}\right)-f\left(u^{\ddagger}\right), x^{k}-u^{\ddagger}\right\rangle \geq 0$. Then, $\left\langle f\left(x^{k}\right)\right.$, $\left.x^{k}-u^{\ddagger}\right\rangle \geq 0$. It equates that $\left\langle f\left(x^{k}\right), t^{k}-u^{\ddagger}\right\rangle \geq\left\langle f\left(x^{k}\right)\right.$, $\left.t^{k}-x^{k}\right\rangle$. Thus,

$$
-2 \tau \zeta^{k} \gamma^{k}\left\langle f\left(x^{k}\right), t^{k}-u^{\ddagger}\right\rangle \leq-2 \tau \zeta^{k} \gamma^{k}\left\langle f\left(x^{k}\right), t^{k}-x^{k}\right\rangle .
$$

On the other hand, combining $t^{k} \in T^{k}$ and Algorithm 1, we obtain

$$
\left\langle\kappa\left(v^{k}, x^{k}\right), t^{k}-x^{k}\right\rangle \leq \gamma^{k}\left\langle f\left(x^{k}\right), t^{k}-x^{k}\right\rangle .
$$

This implies that

$$
-2 \tau \zeta^{k} \gamma^{k}\left\langle f\left(x^{k}\right), t^{k}-u^{\ddagger}\right\rangle \leq-2 \tau \zeta^{k}\left\langle\kappa\left(v^{k}, x^{k}\right), t^{k}-x^{k}\right\rangle .
$$

Hence, we obtain

$$
\begin{aligned}
-2 \tau \zeta^{k} \gamma^{k}\left\langle f\left(x^{k}\right), t^{k}-u^{\ddagger}\right\rangle \leq & -2 \tau \zeta^{k}\left\langle\kappa\left(v^{k}, x^{k}\right), t^{k}-x^{k}\right\rangle \\
\leq & -2 \tau \zeta^{k}\left\langle\kappa\left(v^{k}, x^{k}\right), v^{k}-x^{k}\right\rangle \\
& +2 \tau \zeta^{k}\left\langle\kappa\left(v^{k}, x^{k}\right), v^{k}-t^{k}\right\rangle .
\end{aligned}
$$

Now, we calculate $-2 \tau \zeta^{k}\left\langle\kappa\left(v^{k}, x^{k}\right), v^{k}-x^{k}\right\rangle$ and $2 \tau \zeta^{k}\left\langle\kappa\left(v^{k}, x^{k}\right), v^{k}-t^{k}\right\rangle$ separately. From the definition of $\left\{\zeta^{k}\right\}$, we get

$$
-2 \tau \zeta^{k}\left\langle\kappa\left(v^{k}, x^{k}\right), v^{k}-x^{k}\right\rangle=-2 \tau\left(\zeta^{k}\right)^{2}\left\|\kappa\left(v^{k}, x^{k}\right)\right\|^{2} .
$$

Meanwhile,

$$
\begin{aligned}
2 \tau \zeta^{k}\left\langle\kappa\left(v^{k}, x^{k}\right), v^{k}-t^{k}\right\rangle= & \left\|v^{k}-t^{k}\right\|^{2}+\tau^{2}\left(\zeta^{k}\right)^{2}\left\|\kappa\left(v^{k}, x^{k}\right)\right\|^{2} \\
& -\left\|v^{k}-t^{k}-\tau \zeta^{k} \kappa\left(v^{k}, x^{k}\right)\right\|^{2}
\end{aligned}
$$

This implies that

$$
\begin{aligned}
-2 \tau \zeta^{k} \gamma^{k}\left\langle f\left(x^{k}\right), t^{k}-u^{\ddagger}\right\rangle \leq & -2 \tau\left(\zeta^{k}\right)^{2}\left\|\kappa\left(v^{k}, x^{k}\right)\right\|^{2} \\
& +\left\|v^{k}-t^{k}\right\|^{2}+\tau^{2}\left(\zeta^{k}\right)^{2}\left\|\kappa\left(v^{k}, x^{k}\right)\right\|^{2} \\
-\left\|v^{k}-t^{k}-\tau \zeta^{k} \kappa\left(v^{k}, x^{k}\right)\right\|^{2}= & \left\|v^{k}-t^{k}\right\|^{2}-\left\|v^{k}-t^{k}-\tau \zeta^{k} \kappa\left(v^{k}, x^{k}\right)\right\|^{2} \\
& -\tau(2-\tau)\left(\zeta^{k}\right)^{2}\left\|\kappa\left(v^{k}, x^{k}\right)\right\|^{2} .
\end{aligned}
$$

So, we get

$$
\begin{aligned}
\left\|t^{k}-u^{\ddagger}\right\|^{2} \leq & \left\|v^{k}-u^{\ddagger}\right\|^{2}-\left\|\left(v^{k}-t^{k}\right)-\tau \zeta^{k} \kappa\left(v^{k}, x^{k}\right)\right\|^{2} \\
& -\tau(2-\tau)\left(\zeta^{k}\right)^{2}\left\|\kappa\left(v^{k}, x^{k}\right)\right\|^{2} .
\end{aligned}
$$

This completes the proof.

Theorem 1. The sequence $\left\{u^{k}\right\}$ generated by Algorithm 1 converges strongly to $u^{\ddagger} \in \operatorname{Sol}(\mathrm{S}, f)$.

Proof. We divide the proof into four claims.

Claim 1. We prove the boundedness of the sequences $\left\{u^{k}\right\}$ and $\left\{t^{k}\right\}$. Indeed, from Algorithm 1 and Lemma 6, we get

$$
\begin{aligned}
\left\|u^{k+1}-u^{\ddagger}\right\| & =\left\|\left(1-\theta^{k}-\lambda^{k}\right) v^{k}+\lambda^{k} t^{k}-u^{\ddagger}\right\| \\
& =\left\|\left(1-\theta^{k}-\lambda^{k}\right)\left(v^{k}-u^{\ddagger}\right)+\lambda^{k}\left(t^{k}-u^{\ddagger}\right)-\theta^{k} u^{\ddagger}\right\| \\
& \leq\left\|\left(1-\theta^{k}-\lambda^{k}\right)\left(v^{k}-u^{\ddagger}\right)+\lambda^{k}\left(t^{k}-u^{\ddagger}\right)\right\|+\theta^{k}\left\|u^{\ddagger}\right\| \\
& \leq\left(1-\theta^{k}-\lambda^{k}\right)\left\|v^{k}-u^{\ddagger}\right\|+\lambda^{k}\left\|t^{k}-u^{\ddagger}\right\|+\theta^{k}\left\|u^{\ddagger}\right\|
\end{aligned}
$$


Initialization. Choose $u^{0}, u^{1} \in \mathrm{H}$ arbitrarily.

Step 1. Choose $\rho^{k}$ s.t. $0 \leq \rho^{k} \leq \bar{\rho}^{k}$, where $\bar{\rho}^{k}=\left\{\begin{array}{ll}\min \left\{\rho,\left(\varepsilon^{k} /\left\|u^{k}-u^{k-1}\right\|\right)\right\}, & \text { if } u^{k} \neq u^{k-1} \\ \rho, & \text { otherwise }\end{array}\right.$ Calculate $v^{k}=u^{k}+\rho^{k}\left(u^{k}-u^{k-1}\right)$

Step 2. Calculate $x^{k}=P_{\mathrm{S}}\left(v^{k}-\gamma^{k} f\left(v^{k}\right)\right)$,

where, $\gamma^{k+1}= \begin{cases}\min \left\{\gamma^{k},\left(\sigma\left\|u^{k}-x^{k}\right\| /\left\|f\left(u^{k}\right)-f\left(x^{k}\right)\right\|\right)\right\}, & \text { if } f\left(u^{k}\right) \neq f\left(x^{k}\right) \\ \gamma^{k}, & \text { otherwise }\end{cases}$

Step 3. Construct the half space $T^{k}$ as follows $T^{k}=\left\{z \in H \mid\left\langle v^{k}-\gamma^{k} f\left(v^{k}\right)-x^{k}, z-x^{k}\right\rangle \leq 0\right\}$

Calculate $t^{k}=P_{T^{k}}\left(v^{k}-\tau \zeta^{k} \gamma^{k} f\left(x^{k}\right)\right)$

where $\zeta^{k}=\left(\left\langle v^{k}-x^{k}, \kappa\left(v^{k}, x^{k}\right)\right\rangle /\left\|\kappa\left(v^{k}, x^{k}\right)\right\|^{2}\right)$

and $\kappa\left(v^{k}, x^{k}\right)=v^{k}-x^{k}-\gamma^{k}\left(f\left(v^{k}\right)-f\left(x^{k}\right)\right)$

Step 4. Compute $u^{k+1}=\left(1-\theta^{k}-\lambda^{k}\right) v^{k}+\lambda^{k} t^{k}$

If $x^{k}=v^{k}$, then stop and $x^{k} \in \operatorname{Sol}(S, f)$. Otherwise, set $k:=k+1$ and return to step 1 .

Algorithm 1: Strong convergence algorithm with contractive technique.

$\leq\left(1-\theta^{k}\right)\left\|v^{k}-u^{\ddagger}\right\|-\lambda^{k}\left\|v^{k}-u^{\ddagger}\right\|+\lambda^{k}\left\|v^{k}-u^{\ddagger}\right\|+\theta^{k}\left\|u^{\ddagger}\right\|$

$=\left(1-\theta^{k}\right)\left\|v^{k}-u^{\ddagger}\right\|+\theta^{k}\left\|u^{\ddagger}\right\|$.

Combining Algorithm 1 and (44), we obtain

$$
\begin{aligned}
\left\|u^{k+1}-u^{\ddagger}\right\|= & \left(1-\theta^{k}\right)\left\|v^{k}-u^{\ddagger}\right\|+\theta^{k}\left\|u^{\ddagger}\right\| \\
= & \left(1-\theta^{k}\right)\left\|u^{k}+\rho^{k}\left(u^{k}-u^{k-1}\right)-u^{\ddagger}\right\|+\theta^{k}\left\|u^{\ddagger}\right\| \\
\leq & \left(1-\theta^{k}\right)\left\|u^{k}-u^{\ddagger}\right\|+\rho^{k}\left(1-\theta^{k}\right) \\
& \left\|u^{k}-u^{k-1}\right\|+\theta^{k}\left\|u^{\ddagger}\right\| \\
= & \left(1-\theta^{k}\right)\left\|u^{k}-u^{\ddagger}\right\|+\theta^{k}\left(\varsigma^{k}+\left\|u^{\ddagger}\right\|\right),
\end{aligned}
$$

where

$$
\varsigma^{k}=\left(1-\theta^{k}\right) \frac{\rho^{k}}{\theta^{k}}\left\|u^{k}-u^{k-1}\right\|
$$

Taking into account $\varepsilon^{k}=o\left(\theta^{k}\right)$ and the definition of $\rho^{k}$, we get

$$
\lim _{k \longrightarrow \infty} \varsigma^{k}=0
$$

Then, the sequence $\left\{c^{k}\right\}$ is bounded. Let $M=$ $\sup _{k \geq 1}\left(\varsigma^{k}+\left\|u^{\ddagger}\right\|\right)$. We obtain from (45) that

$$
\begin{aligned}
\left\|u^{k+1}-u^{\ddagger}\right\| & \leq\left(1-\theta^{k}\right)\left\|u^{k}-u^{\ddagger}\right\|+\theta^{k} M \\
& \leq \max \left\{\left\|u^{k}-u^{\ddagger}\right\|, M\right\} .
\end{aligned}
$$

For $\forall k \geq k_{0}$, we have

$$
\left\|u^{k+1}-u^{\ddagger}\right\| \leq \max \left\{\left\|u^{k}-u^{\ddagger}\right\|, M\right\} .
$$

It follows that the sequence $\left\{u^{k}\right\}$ is bounded. Therefore, the sequence $\left\{t^{k}\right\}$ is bounded.

Claim 2. We prove that the following holds:

$$
\begin{aligned}
\left\|u^{k+1}-u^{\ddagger}\right\|^{2} \leq & \left(1-\theta^{k}\right)^{2}\left\|v^{k}-u^{\ddagger}\right\|^{2}-2 \lambda^{k} \theta^{k} \\
& \left\langle v^{k}-t^{k}, u^{k+1}-u^{\ddagger}\right\rangle+2 \theta^{k}\left\langle u^{\ddagger}, u^{k+1}-u^{\ddagger}\right\rangle .
\end{aligned}
$$

Set $z^{k}=\left(1-\lambda^{k}\right) v^{k}+\lambda^{k} t^{k}$. Then, $v^{k}-z^{k}=\lambda^{k}\left(v^{k}-t^{k}\right)$. Therefore,

$$
\begin{aligned}
u^{k+1} & =\left(1-\theta^{k}-\lambda^{k}\right) v^{k}+\lambda^{k} t^{k}=z^{k}-\theta^{k} v^{k} \\
& =\left(1-\theta^{k}\right) z^{k}-\theta^{k}\left(v^{k}-z^{k}\right) \\
& =\left(1-\theta^{k}\right) z^{k}-\theta^{k} \lambda^{k}\left(v^{k}-t^{k}\right) .
\end{aligned}
$$

From Lemma 6, we have

$$
\left\|t^{k}-u^{\ddagger}\right\| \leq\left\|v^{k}-u^{\ddagger}\right\|,
$$

which implies that

$$
\left\|z^{k}-u^{\ddagger}\right\|^{2} \leq\left(1-\lambda^{k}\right)\left\|v^{k}-u^{\ddagger}\right\|^{2}+\lambda^{k}\left\|t^{k}-u^{\ddagger}\right\|^{2} \leq\left\|v^{k}-u^{\ddagger}\right\|^{2} \text {. }
$$

By (7), (51), and (53), we get

$$
\begin{aligned}
\left\|u^{k+1}-u^{\ddagger}\right\|^{2} & =\left\|\left(1-\theta^{k}\right) z^{k}-\theta^{k} \lambda^{k}\left(v^{k}-t^{k}\right)-u^{\ddagger}\right\|^{2} \\
& =\left\|\left(1-\theta^{k}\right)\left(z^{k}-u^{\ddagger}\right)-\theta^{k} \lambda^{k}\left(v^{k}-t^{k}\right)-\theta^{k} u^{\ddagger}\right\|^{2} \\
& \leq\left(1-\theta^{k}\right)^{2}\left\|z^{k}-u^{\ddagger}\right\|^{2}-2 \theta^{k} \lambda^{k}\left\langle v^{k}-t^{k}, u^{k+1}-u^{\ddagger}\right\rangle-2 \theta^{k}\left\langle u^{\ddagger}, u^{k+1}-u^{\ddagger}\right\rangle \\
& \leq\left(1-\theta^{k}\right)^{2}\left\|v^{k}-u^{\ddagger}\right\|^{2}-2 \theta^{k} \lambda^{k}\left\langle v^{k}-t^{k}, u^{k+1}-u^{\ddagger}\right\rangle-2 \theta^{k}\left\langle u^{\ddagger}, u^{k+1}-u^{\ddagger}\right\rangle \\
& =\left(1-\theta^{k}\right)^{2}\left\|v^{k}-u^{\ddagger}\right\|^{2}-2 \theta^{k} \lambda^{k}\left\langle v^{k}-t^{k}, u^{k+1}-u^{\ddagger}\right\rangle+2 \theta^{k}\left\langle-u^{\ddagger}, u^{k+1}-u^{\ddagger}\right\rangle .
\end{aligned}
$$


Claim 3. By (8) and Algorithm 1, we obtain

$$
\begin{aligned}
\left\|v^{k}-u^{\ddagger}\right\|^{2} & =\left\|u^{k}+\rho^{k}\left(u^{k}-u^{k-1}\right)-u^{\ddagger}\right\|^{2} \\
& =\left\|\left(1+\rho^{k}\right)\left(u^{k}-u^{\ddagger}\right)-\rho^{k}\left(u^{k-1}-u^{\ddagger}\right)\right\|^{2} \\
& =\left(1+\rho^{k}\right)\left\|u^{k}-u^{\ddagger}\right\|^{2}-\rho^{k}\left\|u^{k-1}-u^{\ddagger}\right\|^{2}+\rho^{k}\left(1+\rho^{k}\right)\left\|u^{k}-u^{k-1}\right\|^{2} \\
& \leq\left(1+\rho^{k}\right)\left\|u^{k}-u^{\ddagger}\right\|^{2}-\rho^{k}\left\|u^{k-1}-u^{\ddagger}\right\|^{2}+2 \rho^{k}\left\|u^{k}-u^{k-1}\right\|^{2} \\
& =\left\|u^{k}-u^{\ddagger}\right\|^{2}+\rho^{k}\left(\left\|u^{k}-u^{\ddagger}\right\|^{2}-\left\|u^{k-1}-u^{\ddagger}\right\|^{2}\right)+2 \rho^{k}\left\|u^{k}-u^{k-1}\right\|^{2} .
\end{aligned}
$$

Using Lemma (8) and (52), we get

$$
\begin{aligned}
\left\|u^{k+1}-u^{\ddagger}\right\|^{2}= & \left\|\left(1-\theta^{k}-\lambda^{k}\right)\left(v^{k}-u^{\ddagger}\right)+\lambda^{k}\left(t^{k}-u^{\ddagger}\right)+\theta^{k}\left(-u^{\ddagger}\right)\right\|^{2} \\
\leq & \left(1-\theta^{k}-\lambda^{k}\right)\left\|v^{k}-u^{\ddagger}\right\|^{2}+\lambda^{k}\left\|t^{k}-u^{\ddagger}\right\|^{2}+\theta^{k}\left\|u^{\ddagger}\right\|^{2} \\
\leq & \left(1-\theta^{k}-\lambda^{k}\right)\left\|v^{k}-u^{\ddagger}\right\|^{2}+\theta^{k}\left\|u^{\ddagger}\right\|^{2} \\
& +\lambda^{k}\left(\left\|v^{k}-u^{\ddagger}\right\|^{2}-\left\|v^{k}-t^{k}-\tau \zeta^{k} \kappa\left(v^{k}, x^{k}\right)\right\|^{2}\right) \\
& \left.-\tau(2-\tau)\left(\zeta^{k}\right)^{2}\left\|\kappa\left(v^{k}, x^{k}\right)\right\|^{2}\right) \\
\leq & \left(1-\theta^{k}\right)\left\|v^{k}-u^{\ddagger}\right\|^{2}+\theta^{k}\left\|u^{\ddagger}\right\|^{2}-\lambda^{k}\left\|v^{k}-t^{k}-\tau \zeta^{k} \kappa\left(v^{k}, x^{k}\right)\right\|^{2} \\
& \left.-\lambda^{k} \tau(2-\tau)\left(\zeta^{k}\right)^{2}\left\|\kappa\left(v^{k}, x^{k}\right)\right\|^{2}\right) .
\end{aligned}
$$

From (55) and (56), we get

$$
\begin{aligned}
\left\|u^{k+1}-u^{\ddagger}\right\|^{2} \leq & \left(1-\theta^{k}\right)\left\|u^{k}+\rho^{k}\left(u^{k}-u^{k-1}\right)-u^{\ddagger}\right\|^{2}+\theta^{k}\left\|u^{\ddagger}\right\|^{2} \\
& -\lambda^{k}\left\|v^{k}-t^{k}-\tau \zeta^{k} \kappa\left(v^{k}, x^{k}\right)\right\|^{2}-\lambda^{k} \tau(2-\tau)\left(\zeta^{k}\right)^{2}\left\|\kappa\left(v^{k}, x^{k}\right)\right\|^{2} \\
\leq & \left(1-\theta^{k}\right)\left\|u^{k}-u^{\ddagger}\right\|^{2}+\rho^{k}\left(1-\theta^{k}\right)\left(\left\|u^{k}-u^{\ddagger}\right\|^{2}-\left\|u^{k-1}-u^{\ddagger}\right\|^{2}\right) \\
& +2 \rho^{k}\left(1-\theta^{k}\right)\left\|u^{k}-u^{k-1}\right\|^{2}+\theta^{k}\left\|u^{\ddagger}\right\|^{2} \\
& -\lambda^{k}\left\|v^{k}-t^{k}-\tau \zeta^{k} \kappa\left(v^{k}, x^{k}\right)\right\|^{2}-\lambda^{k} \tau(2-\tau)\left(\zeta^{k}\right)^{2}\left\|\kappa\left(v^{k}, x^{k}\right)\right\|^{2} \\
\leq & \left\|u^{k}-u^{\ddagger}\right\|^{2}+\rho^{k}\left(1-\theta^{k}\right)\left(\left\|u^{k}-u^{\ddagger}\right\|^{2}-\left.\left\|u^{k-1}-u^{\ddagger}\right\|\right|^{2}\right) \\
& +2 \rho^{k}\left(1-\theta^{k}\right)\left\|u^{k}-u^{k-1}\right\|^{2}+\theta^{k}\left\|u^{\ddagger}\right\|^{2} \\
& -\lambda^{k}\left\|v^{k}-t^{k}-\tau \zeta^{k} \kappa\left(v^{k}, x^{k}\right)\right\|^{2}-\lambda^{k} \tau(2-\tau)\left(\zeta^{k}\right)^{2}\left\|\kappa\left(v^{k}, x^{k}\right)\right\|^{2} .
\end{aligned}
$$

Claim 4. Next, we will consider two different cases to prove the strong convergence of the sequence $\left\{\left\|u^{k}-u^{\ddagger}\right\|^{2}\right\}$.
Case 1. There exists an $N \in \mathbb{N}$ s.t. $\left\|u^{k+1}-u^{\ddagger}\right\|^{2} \leq\left\|u^{k}-u^{\ddagger}\right\|^{2}, \forall k \geq N$. Obviously, the limit of the sequence $\left\{\left\|u^{k}-u^{\ddagger}\right\|^{2}\right\}$ exists which implies that 
$\lim _{k \longrightarrow \infty}\left\|u^{k+1}-u^{k}\right\|=0$. In (57), taking the limit as $k \longrightarrow \infty$, we deduce

$$
\begin{gathered}
\lim _{k \longrightarrow \infty}\left\|\kappa\left(v^{k}, x^{k}\right)\right\|=0, \\
\lim _{k \rightarrow \infty}\left\|v^{k}-t^{k}-\tau \zeta^{k} \kappa\left(v^{k}, x^{k}\right)\right\|^{2}=0 .
\end{gathered}
$$

On the other hand, we have

$$
\left\|v^{k}-t^{k}\right\| \leq\left\|v^{k}-t^{k}-\tau \zeta^{k} \kappa\left(v^{k}, x^{k}\right)\right\|+\tau \zeta^{k}\left\|\kappa\left(v^{k}, x^{k}\right)\right\| .
$$

So, we have $\lim _{k \rightarrow \infty}\left\|v^{k}-t^{k}\right\|=0$.

Combining Lemma 3 and (58), we obtain

$$
\lim _{k \rightarrow \infty}\left\|v^{k}-x^{k}\right\|=0 \text {. }
$$

Now, we show that $w_{w}\left(u^{k}\right) \subset \operatorname{Sol}(S, f)$. Choose $p^{\ddagger} \in w_{w}\left(u^{k}\right)$. It implies that there exists a subsequence $\left\{u^{n_{k}}\right\}$ of $\left\{u^{k}\right\}$ which converges weakly to $p^{\ddagger}$. Therefore, $v^{n_{k}} \rightarrow p^{\ddagger}$. Due to $\lim _{k \rightarrow \infty}\left\|v^{k}-x^{k}\right\|=0$, we obtain $x^{n_{k}} \rightarrow p^{\ddagger} \in \mathcal{S}$. By Algorithm 1, we have

$$
\left\langle x^{k}-v^{k}+\gamma^{k} f\left(v^{k}\right), u-x^{k}\right\rangle \geq 0, \quad \forall u \in C .
$$

Since $f$ is monotone, we have

$$
\begin{aligned}
0 \leq & \left\langle x^{k}-v^{k}, u-x^{k}\right\rangle+\gamma^{k}\left\langle f\left(v^{k}\right), u-x^{k}\right\rangle \\
= & \left\langle x^{k}-v^{k}, u-x^{k}\right\rangle+\gamma^{k}\left\langle f\left(v^{k}\right), u-v^{k}\right\rangle \\
& +\gamma^{k}\left\langle f\left(v^{k}\right), v^{k}-x^{k}\right\rangle \\
\leq & \left\langle x^{k}-v^{k}, u-x^{k}\right\rangle+\gamma^{k}\left\langle f(u), u-v^{k}\right\rangle \\
& +\gamma^{k}\left\langle f\left(v^{k}\right), v^{k}-x^{k}\right\rangle .
\end{aligned}
$$

Taking the limit in $(63)$ as $k \longrightarrow \infty$, we get

$$
\left\langle f(u), u-p^{\ddagger}\right\rangle \geq 0, \quad \forall u \in C,
$$

which implies that $w_{w}\left(u^{k}\right) \in \operatorname{Sol}(S, f)$.

Set $b^{k}=\left\|u^{k}-u^{\ddagger}\right\|^{2}$ for all $k \geq 0$. By (65) for $q=u^{\ddagger}$, we obtain

$$
\begin{aligned}
b^{k+1} \leq & \left(1-\theta^{k}\right)\left\|v^{k}-q\right\|^{2}+\theta^{k}\left[-2 \lambda^{k}\left\|v^{k}-t^{k}\right\|\left\|u^{k+1}-q\right\|\right. \\
& \left.+2\left\langle-q, u^{k+1}-q\right\rangle\right] .
\end{aligned}
$$

We deduce from Algorithm 1 that

$$
\begin{aligned}
\left\|v^{k}-q\right\|^{2} \leq & \left(\left\|u^{k}-q\right\|+\rho^{k}\left\|u^{k}-u^{k-1}\right\|\right)^{2} \\
= & \left\|u^{k}-q\right\|^{2}+\left(\rho^{k}\right)^{2}\left\|u^{k}-u^{k-1}\right\|^{2} \\
& +2 \rho^{k}\left\|u^{k}-q\right\|\left\|u^{k}-u^{k-1}\right\|
\end{aligned}
$$

$$
\begin{aligned}
& \leq\left\|u^{k}-q\right\|^{2}+\rho^{k}\left\|u^{k}-u^{k-1}\right\|^{2} \\
& \quad+2 \rho^{k}\left\|u^{k}-q\right\|\left\|u^{k}-u^{k-1}\right\| \\
& \leq b^{k}+3 K \rho^{k}\left\|u^{k}-u^{k-1}\right\|,
\end{aligned}
$$

where

$$
K=\sup _{k \geq 1}\left\{\left\|u^{k}-u^{k-1}\right\|,\left\|u^{k}-q\right\|\right\}
$$

By virtue of (65) and (66), we have

$$
b^{k+1} \leq\left(1-\theta^{k}\right) b^{k}+\delta^{k},
$$

where

$$
\begin{gathered}
\delta^{k}=\theta^{k}\left[3 K\left(1-\theta^{k}\right) \frac{\rho^{k}}{\theta^{k}}\left\|u^{k}-u^{k-1}\right\|-2 \lambda^{k}\left\|v^{k}-t^{k}\right\|\right. \\
\left.\left\|u^{k+1}-q\right\|+2\left\langle-q, u^{k+1}-q\right\rangle\right] .
\end{gathered}
$$

So, we get

$$
\limsup _{k \geq 1}\left\langle-q, u^{k+1}-q\right\rangle=\sup _{u^{\ddagger} \in w_{w}}\left(u^{k}\right)\left\langle-q, u^{\ddagger}-q\right\rangle \leq 0 .
$$

From (70), we deduce that $q \in P_{\mathrm{Sol}(\mathrm{S}, f)}(0)$. Combining the property of projection, $\lim _{k \longrightarrow \infty}\left\|v^{k}-t^{k}\right\|^{2}=0$ and $\lim _{k \longrightarrow \infty}\left(\rho^{k} / \theta^{k}\right)\left\|u^{k}-u^{k-1}\right\|=0$, we have $\limsup _{k>1} \delta^{k} \leq 0$. By Lemma 2, we obtain $b^{k}=\left\|u^{k}-u^{\ddagger}\right\|^{2} \longrightarrow 0(k \longrightarrow \infty)$. Therefore, the sequence $\left\{u^{k}\right\}$ converges strongly to $u^{\ddagger}$.

Case 2. There exists a subsequence $\left\{b^{k_{i}}\right\} \subset\left\{b^{k}\right\}_{k \geq \tilde{k}_{0}}$ s.t. $b^{k_{i}} \leq \tilde{b}^{\tilde{k}_{i}+1}$ for $\forall i \geq 0$. From Lemma 2, we can deduce

$$
\begin{gathered}
b^{\gamma(k)} \leq b^{\gamma(k)+1}, \\
b^{k} \leq b^{\gamma(k)+1},
\end{gathered}
$$

for each $k \geq \widetilde{k}_{0}$, where $\gamma(k)=\max \left\{n \in \mathbb{N} \mid \widetilde{k}_{0} \leq n \leq\right.$ $\left.k, b^{n} \leq b^{n+1}\right\}$. Further, the sequence $\{\gamma(k)\}_{k \geq \tilde{k}_{0}}$ is nondecreasing (i.e., $\lim _{k \rightarrow \infty} \gamma(k)=\infty$ ). Let $b^{k}=$ $\left\|u^{k}-u^{\ddagger}\right\|^{2}$. By (71) and Claim 3 for $q=u^{\ddagger}$, we obtain

$$
\begin{aligned}
& \lambda^{\gamma(k)}\left[\left\|v^{\gamma(k)}-t^{\gamma(k)}-\gamma \zeta^{\gamma(k)} d\left(v^{\gamma(k)}, x^{\gamma(k)}\right)\right\|^{2}\right. \\
& \left.\quad+\tau(2-\tau)\left(\zeta^{\gamma(k)}\right)^{2}\left\|d\left(v^{\gamma(k)}, x^{\gamma(k)}\right)\right\|^{2}\right] \\
& \leq \rho^{\gamma(k)}\left(1-\theta^{\gamma(k)}\right)\left(b^{\gamma(k)}-b^{\gamma(n)-1}\right) \\
& \quad+2 \rho^{\gamma(k)}\left(1-\theta^{\gamma(k)}\right)\left\|u^{\gamma(k)}-u^{\gamma(n)-1}\right\|^{2}+\theta^{\gamma(k)}\|q\|^{2} .
\end{aligned}
$$

We deduce from the definition of $b^{k}$ that 


$$
\begin{aligned}
b^{\gamma(k)}-b^{\gamma(k)-1}= & \left\|u^{\gamma(k)}-q\right\|^{2}-\left\|u^{\gamma(k)-1}-q\right\|^{2} \\
= & \left(\left\|u^{\gamma(k)}-q\right\|-\left\|u^{\gamma(k)-1}-q\right\|\right) \\
& \left(\left\|u^{\gamma(k)}-q\right\|+\left\|u^{\gamma(k)-1}-q\right\|\right) \\
\leq & \left\|u^{\gamma(k)}-u^{\gamma(k)-1}\right\|\left(\left\|u^{\gamma(k)}-q\right\|+\left\|u^{\gamma(k)-1}-q\right\|\right) .
\end{aligned}
$$

Combining (72) and (73), we have

$$
\begin{aligned}
& \lambda^{\gamma(k)}\left[\left\|v^{\gamma(k)}-t^{\gamma(k)}-\gamma \zeta^{\gamma(k)} d\left(v^{\gamma(k)}, u^{\gamma(k)}\right)\right\|^{2}\right. \\
& \left.\quad+\tau(2-\tau)\left(\zeta^{\gamma(k)}\right)^{2}\left\|d\left(v^{\gamma(k)}, u^{\gamma(k)}\right)\right\|^{2}\right] \\
& \leq \rho^{\gamma(k)}\left(1-\theta^{\gamma(k)}\right)\left[\left\|u^{\gamma(k)}-u^{\gamma(k)-1}\right\|\left(\left\|u^{\gamma(k)}-q\right\|-\left\|u^{\gamma(k)-1}-q\right\|\right)\right] \\
& \quad+2 \rho^{\gamma(k)}\left(1-\theta^{\gamma(k)}\right)\left\|u^{\gamma(k)}-u^{\gamma(k)-1}\right\|^{2}+\theta^{\gamma(k)}\|q\|^{2} .
\end{aligned}
$$

Similarly, we have $\rho^{\gamma(k)}\left(1-\theta^{\gamma(k)}\right)\left\|u^{\gamma(k)}-u^{\gamma(k)-1}\right\| \longrightarrow 0$. It follows that

$$
\begin{aligned}
w_{w}\left(u^{\gamma(k)}\right) & \subset \operatorname{Sol}(\mathrm{S}, f), \\
\lim _{k \rightarrow \infty}\left\|t^{\gamma(k)}-u^{\gamma(k)}\right\|^{2} & =\lim _{k \longrightarrow \infty}\left\|t^{\gamma(k)}-v^{\gamma(k)}\right\|^{2}=0,
\end{aligned}
$$

and

$$
\begin{aligned}
b^{\gamma(k)+1} \leq & \left(1-\theta^{\gamma(k)}\right) b^{\gamma(k)}+\theta^{\gamma(k)} \\
& {\left[3 K\left(1-\theta^{\gamma(k)}\right) \frac{\rho^{\gamma(k)}}{\theta^{\gamma(k)}}\left\|u^{\gamma(k)}-u^{\gamma(k)-1}\right\|\right.} \\
& -2 \lambda^{\gamma(k)}\left\|v^{\gamma(k)}-t^{\gamma(k)}\right\|\left\|u^{\gamma(k)+1}-q\right\|+2\left\langle-q, u^{\gamma(k)+1}-q\right\rangle .
\end{aligned}
$$

Since $b^{\gamma(k)} \leq b^{\gamma(k)+1}$ and $\theta^{\gamma(k)}>0$, from (76), we have

$$
\begin{aligned}
b^{\gamma(k)} \leq & 3 K\left(1-\theta^{\gamma(k)}\right) \frac{\rho^{\gamma(k)}}{\theta^{\gamma(k)}}\left\|u^{\gamma(k)}-u^{\gamma(k)-1}\right\| \\
& -2 \lambda^{\gamma(k)}\left\|v^{\gamma(k)}-t^{\gamma(k)}\right\|\left\|u^{\gamma(k)+1}-q\right\|+2\left\langle-q, u^{\gamma(k)+1}-q\right\rangle .
\end{aligned}
$$

Since $q \in P_{\mathrm{Sol}(\mathrm{S}, f)}(0)$ and $w_{w}\left(u^{\gamma(k)}\right) \subset \operatorname{Sol}(\mathrm{S}, f)$, we have $\limsup _{k \rightarrow \infty}\left\langle-q, u^{\gamma(k)+1}-q\right\rangle=\sup _{k \rightarrow \infty}\left\langle-q, u^{\ddagger}-q\right\rangle \leq 0$. By (75), (77), and $\left(\rho^{\gamma(k)} / \theta^{\gamma(k)}\right)\left\|u^{\gamma(k)}-u^{\gamma(k)-1}\right\| \longrightarrow 0$, we get

$$
\limsup _{k \longrightarrow \infty} b^{\gamma(k)} \leq 2 \sup _{q \in w_{w}}\left(u^{\gamma(k)}\right)\left\langle-q, u^{\ddagger}-q\right\rangle \leq 0 .
$$

It follows from (76) that

$$
\begin{aligned}
\underset{k \longrightarrow \infty}{\limsup } b^{\gamma(k)+1} \leq 0 & \leq 0 \\
\text { or } \lim _{k \rightarrow \infty} b^{\gamma(k)+1} & =0 .
\end{aligned}
$$

Hence, $\lim _{k \longrightarrow \infty} b^{k}=0$. Therefore, the sequence $\left\{u^{k}\right\}$ converges strongly to $u^{\ddagger}$. This completes the proof.

Suppose that $g: \mathrm{H} \longrightarrow \mathrm{H}$ is a $\rho$-contractive operator. Next, we propose an iterative algorithm with viscosity item.

Theorem 2. The sequence $\left\{u^{k}\right\}$ generated by Algorithm 1 converges strongly to $u^{\ddagger}=P_{S o l(\mathrm{~S}, f)} g\left(u^{\ddagger}\right)$.

Proof. We divide the proof into 4 claims.

Claim 1. We prove the boundedness of the sequences $\left\{g\left(v^{k}\right)\right\},\left\{x^{k}\right\}$ and $\left\{t^{k}\right\}$. From Algorithm 1, we get

$$
\begin{aligned}
\left\|v^{k}-u^{\ddagger}\right\| & =\left\|u^{k}-\rho^{k}\left(u^{k}-u^{k-1}\right)-u^{\ddagger}\right\| \\
& \leq\left\|u^{k}-u^{\ddagger}\right\|+\rho^{k}\left\|u^{k}-u^{k-1}\right\| \\
& =\left\|u^{k}-u^{\ddagger}\right\|+\theta^{k} \frac{\rho^{k}}{\theta^{k}}\left\|u^{k}-u^{k-1}\right\| .
\end{aligned}
$$

From Algorithm 1, we obtain $\left(\rho^{k} / \theta^{k}\right) \| u^{k}$ $u^{k-1} \| \longrightarrow 0,(k \longrightarrow \infty)$. Then, $\exists M_{1}>0$ s.t.

$$
\frac{\rho^{k}}{\theta^{k}}\left\|u^{k}-u^{k-1}\right\| \leq M_{1}, \quad \forall k>0 .
$$

By Algorithm 1 and (81), we have

$$
\begin{aligned}
\left\|u^{k+1}-u^{\ddagger}\right\| & =\left\|\theta^{k} g\left(v^{k}\right)+\left(1-\theta^{k}\right) t^{k}-u^{\ddagger}\right\| \\
& =\left\|\theta^{k}\left(g\left(v^{k}\right)-u^{\ddagger}\right)+\left(1-\theta^{k}\right)\left(t^{k}-u^{\ddagger}\right)\right\| \\
& \leq \theta^{k}\left\|g\left(v^{k}\right)-u^{\ddagger}\right\|+\left(1-\theta^{k}\right)\left\|t^{k}-u^{\ddagger}\right\| \\
& \leq \theta^{k}\left\|g\left(v^{k}\right)-g\left(u^{\ddagger}\right)\right\|+\theta^{k}\left\|g\left(u^{\ddagger}\right)-u^{\ddagger}\right\|+\left(1-\theta^{k}\right)\left\|t^{k}-u^{\ddagger}\right\| \\
& \leq \theta^{k} \rho\left\|v^{k}-u^{\ddagger}\right\|+\theta^{k}\left\|g\left(u^{\ddagger}\right)-u^{\ddagger}\right\|+\left(1-\theta^{k}\right)\left\|v^{k}-u^{\ddagger}\right\|
\end{aligned}
$$


Initialization. Choose $u^{0}, u^{1} \in \mathrm{H}$ arbitrarily.

Step 1. Choose $\rho^{k}$ s.t. $0 \leq \rho^{k} \leq \bar{\rho}^{k}$, where $\bar{\rho}^{k}$
Calculate $v^{k}=u^{k}+\rho^{k}\left(u^{k}-u^{k-1}\right)$ $\begin{cases}\min \left\{\rho,\left(\varepsilon^{k} /\left\|u^{k}-u^{k-1}\right\|\right)\right\} & \text { if } u^{k} \neq u^{k-1} \\ \rho & \text { otherwise }\end{cases}$

Step 2. Calculate $x^{k}=P_{\mathrm{S}}\left(v^{k}-\gamma^{k} f\left(v^{k}\right)\right)$,

where $\gamma^{k+1}= \begin{cases}\min \left\{\gamma^{k},\left(\sigma\left\|u^{k}-x^{k}\right\| /\left\|f u^{k}-f x^{k}\right\|\right)\right\} & \text { iff } u^{k} \neq f x^{k} \\ \gamma^{k} & \text { otherwise }\end{cases}$

Step 3. Construct the half space $T^{k}$ as follows $T^{k}=\left\{z \in \mathrm{O} \in \mid\left\langle v^{k}-\gamma^{k} f v^{k}-x^{k}, z-x^{k}\right\rangle \leq 0\right\}$.

Calculate $t^{k}=P_{T^{k}}\left(v^{k}-\tau \zeta^{k} \gamma^{k} f\left(x^{k}\right)\right)$,

where $\zeta^{k}=\left(\left\langle v^{k}-x^{k}, \kappa\left(v^{k}, x^{k}\right)\right\rangle /\left\|\kappa\left(v^{k}, x^{k}\right)\right\|^{2}\right)$

and $\kappa\left(v^{k}, x^{k}\right)=\left(v^{k}, x^{k}\right)-\gamma^{k}\left(f\left(v^{k}\right)-f\left(x^{k}\right)\right)$.

Step 4. Compute $u^{k+1}=\theta^{k} g\left(v^{k}\right)+\left(1-\theta^{k}\right) t^{k}$.

If $x^{k}=v^{k}$, then stop and $x^{k} \in \operatorname{Sol}(\mathrm{S}, f)$. Otherwise, set $k:=k+1$ and return to step 1 .

Algorithm 2: Strong convergence algorithm with viscosity term.

$$
\begin{aligned}
& \leq\left(1-(1-\rho) \theta^{k}\right)\left\|v^{k}-u^{\ddagger}\right\|+\theta^{k}\left\|g\left(u^{\ddagger}\right)-u^{\ddagger}\right\| \\
& \leq\left(1-(1-\rho) \theta^{k}\right)\left\|u^{k}+\rho^{k}\left(u^{k}-u^{k-1}\right)-u^{\ddagger}\right\|+\theta^{k}\left\|g\left(u^{\ddagger}\right)-u^{\ddagger}\right\| \\
& \leq\left(1-(1-\rho) \theta^{k}\right)\left\|u^{k}-u^{\ddagger}\right\|+\left(1-(1-\rho) \theta^{k}\right) \rho^{k}\left\|u^{k}-u^{k-1}\right\|+\theta^{k}\left\|g\left(u^{\ddagger}\right)-u^{\ddagger}\right\| \\
& \leq\left(1-(1-\rho) \theta^{k}\right)\left\|u^{k}-u^{\ddagger}\right\|+\left(1-(1-\rho) \theta^{k}\right) \theta^{k} \frac{\rho^{k}}{\theta^{k}}\left\|u^{k}-u^{k-1}\right\|+\theta^{k}\left\|g\left(u^{\ddagger}\right)-u^{\ddagger}\right\| .
\end{aligned}
$$

From (81) and (82), we have

$$
\begin{aligned}
\left\|u^{k+1}-u^{\ddagger}\right\| \leq & \left(1-(1-\rho) \theta^{k}\right)\left\|u^{k}-u^{\ddagger}\right\|+\left(1-(1-\rho) \theta^{k}\right) \theta^{k} \frac{\rho^{k}}{\theta^{k}}\left\|u^{k}-u^{k-1}\right\|+\theta^{k}\left\|g\left(u^{\ddagger}\right)-u^{\ddagger}\right\| \\
\leq & \left(1-(1-\rho) \theta^{k}\right)\left\|u^{k}-u^{\ddagger}\right\|+\left(1-(1-\rho) \theta^{k}\right) \theta^{k} M_{1}+\theta^{k}\left\|g\left(u^{\ddagger}\right)-u^{\ddagger}\right\| \leq\left(1-(1-\rho) \theta^{k}\right)\left\|u^{k}-u^{\ddagger}\right\| \\
& +(1-\rho) \theta^{k} \frac{\left(1-(1-\rho) \theta^{k}\right) M_{1}+\left\|g\left(u^{\ddagger}\right)-u^{\ddagger}\right\|}{1-\rho} \\
\leq & \left(1-(1-\rho) \theta^{k}\right)\left\|u^{k}-u^{\ddagger}\right\|+(1-\rho) \theta^{k} \frac{M_{1}+\left\|g\left(u^{\ddagger}\right)-u^{\ddagger}\right\|}{1-\rho} \\
\leq & \max \left\{\left\|u^{k}-u^{\ddagger}\right\|, \frac{M_{1}+\left\|g\left(u^{\ddagger}\right)-u^{\ddagger}\right\|}{1-\rho}\right\} \leq \cdots \leq \max \left\{\left\|u^{k}-u^{\ddagger}\right\|, \frac{M_{1}+\left\|g\left(u^{\ddagger}\right)-u^{\ddagger}\right\|}{1-\rho}\right\} .
\end{aligned}
$$

It is obvious that the sequence $\left\{u^{k}\right\}$ is bounded. Furthermore, the sequences $\left\{g\left(v^{k}\right)\right\},\left\{x^{k}\right\}$ and $\left\{t^{k}\right\}$ are bounded.

$$
\begin{aligned}
\left\|u^{k+1}-u^{\ddagger}\right\|^{2} & =\left\|\theta^{k} g\left(v^{k}\right)+\left(1-\theta^{k}\right) t^{k}-u^{\ddagger}\right\|^{2} \\
& =\left\|\theta^{k}\left(g\left(v^{k}\right)-g\left(u^{\ddagger}\right)\right)+\left(1-\theta^{k}\right)\left(t^{k}-u^{\ddagger}\right)+\theta^{k}\left(g\left(u^{\ddagger}\right)-u^{\ddagger}\right)\right\|^{2} \\
& \leq\left\|\theta^{k}\left(g\left(v^{k}\right)-g\left(u^{\ddagger}\right)\right)+\left(1-\theta^{k}\right)\left(t^{k}-u^{\ddagger}\right)\right\|^{2}
\end{aligned}
$$




$$
\begin{aligned}
& +2 \theta^{k}\left\langle g\left(u^{\ddagger}\right)-u^{\ddagger}, u^{k+1}-u^{\ddagger}\right\rangle \leq \theta^{k}\left\|g\left(v^{k}\right)-g\left(u^{\ddagger}\right)\right\|^{2}+\left(1-\theta^{k}\right)\left\|t^{k}-u^{\ddagger}\right\|^{2} \\
& +2 \theta^{k}\left\langle g\left(u^{\ddagger}\right)-u^{\ddagger}, u^{k+1}-u^{\ddagger}\right\rangle \leq \theta^{k} \rho\left\|v^{k}-u^{\ddagger}\right\|^{2}+\left(1-\theta^{k}\right)\left\|v^{k}-u^{\ddagger}\right\|^{2}+2 \theta^{k}\left\langle g\left(u^{\ddagger}\right)-u^{\ddagger}, u^{k+1}-u^{\ddagger}\right\rangle \\
= & \left(1-(1-\rho) \theta^{k}\right)\left\|v^{k}-u^{\ddagger}\right\|^{2}+2 \theta^{k}\left\langle g\left(u^{\ddagger}\right)-u^{\ddagger}, u^{k+1}-u^{\ddagger}\right\rangle .
\end{aligned}
$$

Claim 3. By (8) and (55), we obtain

$$
\begin{aligned}
\left\|u^{k+1}-u^{\ddagger}\right\|^{2}= & \left\|\theta^{k}\left(g\left(v^{k}\right)-u^{\ddagger}\right)+\left(1-\theta^{k}\right)\left(t^{k}-u^{\ddagger}\right)\right\|^{2} \\
\leq & \theta^{k}\left\|g\left(v^{k}\right)-u^{\ddagger}\right\|^{2}+\left(1-\theta^{k}\right)\left\|t^{k}-u^{\ddagger}\right\|^{2}-\theta^{k}\left(1-\theta^{k}\right)\left\|g\left(v^{k}\right)-t^{k}\right\|^{2} \\
\leq & \theta^{k}\left\|g\left(v^{k}\right)-u^{\ddagger}\right\|^{2}+\left(1-\theta^{k}\right)\left\|t^{k}-u^{\ddagger}\right\|^{2} \leq \theta^{k}\left\|g\left(v^{k}\right)-u^{\ddagger}\right\|^{2}+\left(1-\theta^{k}\right)\left\|v^{k}-u^{\ddagger}\right\|^{2} \\
& -\left(1-\theta^{k}\right)\left\|v^{k}-t^{k}-\tau \zeta^{k} \kappa\left(v^{k}, x^{k}\right)\right\|^{2}-\left(1-\theta^{k}\right) \tau(2-\tau)\left(\zeta^{k}\right)^{2}\left\|\kappa\left(v^{k}, x^{k}\right)\right\|^{2} .
\end{aligned}
$$

From (85) and (55), we obtain

$$
\begin{aligned}
\left\|u^{k+1}-u^{\ddagger}\right\|^{2} \leq & \theta^{k}\left\|g\left(v^{k}\right)-u^{\ddagger}\right\|^{2}+\left(1-\theta^{k}\right)\left\|u^{k}-u^{\ddagger}\right\|^{2} \\
& +\left(1-\theta^{k}\right) \rho^{k}\left(\left\|u^{k}-u^{\ddagger}\right\|^{2}-\left\|u^{k-1}-u^{\ddagger}\right\|^{2}\right) \\
& +2\left(1-\theta^{k}\right) \rho^{k}\left\|u^{k}-u^{k-1}\right\|^{2}-\left(1-\theta^{k}\right)\left\|\left(v^{k}-t^{k}\right)-\tau \zeta^{k} \kappa\left(v^{k}, x^{k}\right)\right\|^{2} \\
& -\left(1-\theta^{k}\right) \tau(2-\tau)\left(\zeta^{k}\right)^{2}\left\|\kappa\left(v^{k}, x^{k}\right)\right\|^{2} \\
\leq & \theta^{k}\left\|g\left(v^{k}\right)-u^{\ddagger}\right\|^{2}+\left\|u^{k}-u^{\ddagger}\right\|^{2}+\left(1-\theta^{k}\right) \rho^{k}\left(\left\|u^{k}-u^{\ddagger}\right\|^{2}-\left\|u^{k-1}-u^{\ddagger}\right\|^{2}\right) \\
& +2\left(1-\theta^{k}\right) \rho^{k}\left\|u^{k}-u^{k-1}\right\|^{2}-\left(1-\theta^{k}\right)\left\|v^{k}-t^{k}-\tau \zeta^{k} \kappa\left(v^{k}, x^{k}\right)\right\|^{2}-\left(1-\theta^{k}\right) \tau(2-\tau)\left(\zeta^{k}\right)^{2}\left\|\kappa\left(v^{k}, x^{k}\right)\right\|^{2} .
\end{aligned}
$$

This implies that

$$
\begin{aligned}
& \left(1-\theta^{k}\right)\left\|v^{k}-t^{k}-\tau \zeta^{k} \kappa\left(v^{k}, x^{k}\right)\right\|^{2}+\left(1-\theta^{k}\right) \tau(2-\tau)\left(\zeta^{k}\right)^{2}\left\|\kappa\left(v^{k}, x^{k}\right)\right\|^{2} \\
& \quad-\left(1-\theta^{k}\right) \rho^{k}\left(\left\|u^{k}-u^{\ddagger}\right\|^{2}-\left\|u^{k-1}-u^{\ddagger}\right\|^{2}\right)-2\left(1-\theta^{k}\right) \rho^{k}\left\|u^{k}-u^{k-1}\right\|^{2} \\
& \leq\left\|u^{k}-u^{\ddagger}\right\|^{2}-\left\|u^{k+1}-u^{\ddagger}\right\|^{2}+\theta^{k}\left\|g\left(v^{k}\right)-u^{\ddagger}\right\|^{2} .
\end{aligned}
$$

Claim 4. According to Claim 3, we can see that there are two possible cases.

Case 1. There exists an $N \in \mathbb{N}$, s.t. $\left\|u^{k+1}-u^{\ddagger}\right\|^{2}$ $\leq\left\|u^{k}-u^{\ddagger}\right\|^{2}$ for $\forall k>N$. It follows that $\lim _{k \rightarrow \infty} \| u^{k}-$ $u^{\ddagger} \|$ exists. From (86) and $\lim _{k \longrightarrow \infty} \theta^{k}=0$, we have

$$
\begin{array}{r}
\lim _{k \longrightarrow \infty}\left\|\kappa\left(v^{k}, x^{k}\right)\right\|=0, \\
\lim _{k \longrightarrow \infty}\left\|v^{k}-t^{k}-\tau \zeta^{k} \kappa\left(v^{k}, x^{k}\right)\right\|=0 .
\end{array}
$$




$$
\left\|v^{k}-t^{k}\right\|^{2} \leq\left\|v^{k}-t^{k}-\tau \zeta^{k} \kappa\left(v^{k}, x^{k}\right)\right\|+\left\|\tau \zeta^{k} \kappa\left(v^{k}, x^{k}\right)\right\| .
$$

So,

$$
\lim _{k \longrightarrow \infty}\left\|v^{k}-t^{k}\right\|=0 .
$$

Similarly, we can obtain

$$
w_{w}\left(u^{k}\right) \subset \operatorname{Sol}(\mathrm{S}, f) .
$$

Set $b^{k}=\left\|u^{k}-q\right\|^{2}$ for all $k \geq 0$. By (84) for $q=u^{\ddagger}$, we get

$$
\begin{aligned}
b^{k+1} \leq & \left(1-(1-\rho) \theta^{k}\right)\left\|v^{k}-u^{\ddagger}\right\|^{2} \\
& +2 \theta^{k}\left\langle g\left(u^{\ddagger}\right)-u^{\ddagger}, u^{k+1}-u^{\ddagger}\right\rangle .
\end{aligned}
$$

It follows from (66) and (92) that

$$
b^{k+1} \leq\left(1-(1-\rho) \theta^{k}\right) b^{k}+\delta^{k},
$$

where

$$
\begin{aligned}
\delta^{k}= & 3 K\left(1-(1-\rho) \theta^{k}\right) \rho^{k}\left\|u^{k}-u^{k-1}\right\| \\
& +2 \theta^{k}\left\langle g(q)-q, u^{k+1}-q\right\rangle .
\end{aligned}
$$

Then,

$$
\begin{gathered}
\underset{k \geq 1}{\limsup }\left\langle g(q)-q, u^{k+1}-q\right\rangle=\underset{u^{\ddagger} \in w_{w}}{\limsup } \\
\left(u^{k}\right)\left\langle g(q)-q, u^{\ddagger}-q\right\rangle \leq 0 .
\end{gathered}
$$

Hence, we deduce $q \in P_{\mathrm{Sol}(\mathrm{S}, f)}(0)$. Combining the property of projection, $\lim _{k \longrightarrow \infty}\left\|v^{k}-t^{k}\right\|^{2}=0$ and $\lim _{k \rightarrow \infty}\left(\rho^{k} / \theta^{k}\right)\left\|u^{k}-u^{k-1}\right\|=0$, we have $\limsup _{k \geq 1}$ $\delta^{k} \leq 0$. By Lemma 2, we obtain $b^{k}=\left\|u^{k}-u^{\ddagger}\right\|^{2}$ $\longrightarrow 0(k \longrightarrow \infty)$. Therefore, the sequence $\left\{u^{k}\right\}$ converges strongly to $u^{\ddagger}$.

Case 2. There exists a subsequence $\left\{b^{k_{i}}\right\} \subset\left\{b^{k}\right\}_{k>\tilde{k}}$, s.t. $b^{k_{i}} \leq b^{k_{i}+1}$ for each $i \geq 0$. From Lemma 1, we deduce that

$$
\begin{gathered}
b^{\gamma(k)} \leq b^{\gamma(k)+1}, \\
b^{k} \leq b^{\gamma(k)+1},
\end{gathered}
$$

for all $\quad k \geq \widetilde{k}_{0}$, where $\quad \gamma(k)=\max \left\{n \in \mathbb{N} \mid \widetilde{k}_{0} \leq\right.$ $\left.n \leq k, b^{n} \leq b^{n+1}\right\}$. Therefore, the sequence $\{\gamma(k)\}_{k \geq \tilde{k}_{0}}$ is nondecreasing (i.e., $\lim _{k \longrightarrow \infty} \gamma(k)=\infty$ ). By (96) and Claim 3 for $q=u^{\ddagger}$, we obtain

$$
\begin{gathered}
\left(1-\theta^{\gamma(k)}\right)\left\|\left(v^{\gamma(k)}-t^{\gamma(k)}\right)-\tau \zeta^{(\gamma(k))} \kappa\left(v^{\gamma(k)}, x^{\gamma(k)}\right)\right\|^{2} \\
+\left(1-\theta^{\gamma(k)}\right) \tau(2-\tau)\left(\zeta^{\gamma(k)}\right)^{2}\left\|\kappa\left(v^{\gamma(k)}, x^{\gamma(k)}\right)\right\|^{2}
\end{gathered}
$$

$$
\begin{aligned}
\leq & \left\|u^{\gamma(k)}-q\right\|^{2}-\left\|u^{\gamma(n)+1}-u^{\ddagger}\right\|^{2}+\theta^{\gamma(k)}\left\|g\left(v^{\gamma(k)}\right)-q\right\|^{2} \\
& +\left(1-\theta^{\gamma(k)}\right) \rho^{\gamma(k)}\left(b^{\gamma(k)}-b^{\gamma(k)-1}\right)+2\left(1-\theta^{\gamma(k)}\right) \rho^{\gamma(k)} \\
& \left\|u^{\gamma(k)}-u^{\gamma(k)-1}\right\|^{2} .
\end{aligned}
$$

From (73) and (97), we get

$$
\begin{aligned}
& \left(1-\theta^{\gamma(k)}\right)\left\|\left(v^{\gamma(k)}-t^{\gamma(k)}\right)-\tau \zeta^{\gamma(k)} \kappa\left(v^{\gamma(k)}, x^{\gamma(k)}\right)\right\|^{2} \\
& \quad+\left(1-\theta^{\gamma(k)}\right) \tau(2-\tau)\left(\zeta^{\gamma(k)}\right)^{2}\left\|\kappa\left(v^{\gamma(k)}, x^{\gamma(k)}\right)\right\|^{2} \\
& \leq\left\|u^{\gamma(k)}-u^{\ddagger}\right\|^{2}-\left\|u^{\gamma(k)+1}-u^{\ddagger}\right\|^{2}+\theta^{\gamma(k)}\left\|g\left(v^{\gamma(k)}\right)-u^{\ddagger}\right\|^{2} \\
& \quad+2\left(1-\theta^{\gamma(k)}\right) \rho^{\gamma(k)}\left\|u^{\gamma(k)}-u^{\gamma(n)-1}\right\|^{2}+\left(1-\theta^{\gamma(k)}\right) \rho^{\gamma(k)} \\
& \left\|u^{\gamma(k)}-u^{\gamma(n)-1}\right\|\left(\left\|u^{\gamma(k)}-q\right\|+\left\|u^{\gamma(n)-1}-q\right\|\right) .
\end{aligned}
$$

Using Claim 1 and (98), we have $\lim _{k \longrightarrow \infty}\left(1-\theta^{\gamma(k)}\right) \rho^{\gamma(k)}\left\|u^{\gamma(k)}-u^{\gamma(k)-1}\right\|=0$. Therefore,

$$
\begin{aligned}
w_{w}\left(u^{\gamma(k)}\right) \subset & \operatorname{Sol}(\mathrm{S}, f), \\
\lim _{k \longrightarrow \infty}\left\|t^{\gamma(k)}-u^{\gamma(k)}\right\|^{2}= & \lim _{k \longrightarrow \infty}\left\|t^{\gamma(k)}-v^{\gamma(k)}\right\|^{2}=0, \\
b^{\gamma(k)+1} \leq & \left(1-(1-\rho) \theta^{\gamma(k)}\right) b^{\gamma(k)}+3 K \\
& \left(1-(1-\rho) \theta^{\gamma(k)}\right) \rho^{\gamma(k)}\left\|u^{\gamma(k)}-u^{\gamma(k)-1}\right\| \\
& +2 \theta^{\gamma(k)}\left\langle g(q)-q, u^{\gamma(k)+1}-q\right\rangle .
\end{aligned}
$$

Since $b^{\gamma(k)} \leq b^{\gamma(k)+1}$ and $b^{\gamma(k)} \geq 0$, we receive

$$
\begin{aligned}
b^{\gamma(k)} \leq & 3 K\left(1-(1-\rho) \theta^{\gamma(k)}\right) \rho^{\gamma(k)}\left\|u^{\gamma(k)}-u^{\gamma(k)-1}\right\| \\
& +2 \theta^{\gamma(k)}\left\langle g(q)-q, u^{\gamma(k)+1}-q\right\rangle .
\end{aligned}
$$

Note that $q \in P_{\mathrm{Sol}(\mathrm{S}, f)}(0)$ and $w_{w}\left(u^{\gamma(k)}\right) \subset \operatorname{Sol}(\mathrm{S}, f)$. By the property of projection, we have

$$
\begin{aligned}
& \limsup _{k \rightarrow \infty}\left\langle g(q)-q, u^{\gamma(k)+1}-q\right\rangle=\sup _{u^{\ddagger} \in w_{w}}\left(u^{\gamma(k)}\right) \\
& \left\langle g(q)-q, u^{\ddagger}-q\right\rangle \leq 0 .
\end{aligned}
$$

Since $\left(1-\theta^{\gamma(k)}\right) \rho^{\gamma(k)}\left\|u^{\gamma(k)}-u^{\gamma(n)-1}\right\| \longrightarrow 0$, we deduce

$$
\limsup _{k \longrightarrow \infty} a_{\gamma(k)} \leq 2 \sup _{u^{\ddagger} \in w_{w}}\left(x_{\gamma(k)}\right)\left\langle g(q)-q, u^{\ddagger}-q\right\rangle \leq 0 .
$$

So, $\limsup _{k \longrightarrow \infty} b^{\gamma(k)+1} \leq 0$ or $\lim _{k \longrightarrow \infty} b^{\gamma(k)+1}=0$. Hence, $\lim _{k \rightarrow \infty} b^{k}=0$ which implies that the sequence $\left\{u^{k}\right\}$ converges strongly to $u^{\ddagger}$. This completes the proof. 


\section{Data Availability}

No data were used to support this study.

\section{Conflicts of Interest}

The authors declare that they have no conflicts of interest.

\section{Authors' Contributions}

All authors contributed equally and significantly in writing this article. All authors read and approved the final manuscript.

\section{Acknowledgments}

Danfeng $\mathrm{Wu}$ was supported by Postgraduate Innovation Project of North Minzu University (YCX21155); Li-Jun Zhu was supported by the National Natural Science Foundation of China (61362033) and the Natural Science Foundation of Ningxia Province (NZ17015); and Zhuang Shan was supported by Postgraduate Innovation Project of North Minzu University (YCX21153).

\section{References}

[1] H. H. Bauschke and P. L. Combettes, Convex Analysis and Monotone Operator Theory in Hilbert Spaces, Springer, Berlin, Germany, 2011.

[2] C. L. Byrne, "A unified treatment of some iterative algorithms in signal processing and image reconstruction," Inverse Problems, vol. 20, no. 1, pp. 1-30, 2004.

[3] Y. Yao, L. Leng, M. Postolache, and X. Zheng, "Mann-type iteration method for solving the split common fixed point problem," Journal of Nonlinear and Convex Analysis, vol. 18, pp. 875-882, 2017.

[4] M. Aslam Noor, "Some developments in general variational inequalities," Applied Mathematics and Computation, vol. 152, no. 1, pp. 199-277, 2004.

[5] M. Fukushima, "A relaxed projection method for variational inequalities," Mathematical Programming, vol. 35, no. 1, pp. 58-70, 1986.

[6] Y. Yao, Y.-C. Liou, and M. Postolache, "Self-adaptive algorithms for the split problem of the demicontractive operators," Optimization, vol. 67, no. 9, pp. 1309-1319, 2018.

[7] K. Rajendra Prasad, M. Khuddush, and D. Leela, "Existence of solutions for fractional order BVPs by mixed monotone ternary operator with perturbation on Banach spaces," Journal of Advanced Mathematical Studies, vol. 14, pp. 109$125,2021$.

[8] R. W. Cottle and J. C. Yao, "Pseudo-monotone complementarity problems in Hilbert space," Journal of Optimization Theory and Applications, vol. 75, no. 2, pp. 281-295, 1992.

[9] Y. Yao, M. Postolache, and Z. Zhu, "Gradient methods with selection technique for the multiple-sets split feasibility problem," Optimization, vol. 69, no. 2, pp. 269-281, 2020.

[10] F. Facchinei and J. S. Pang, "Finite-dimensional variational inequalities and complementarity problems," Springer Series in Operations Research, Vol. 1, Springer, New York, NY, USA, 2003.

[11] Y. Censor, A. Gibali, and S. Reich, "Extensions of Korpelevich's extragradient method for the variational inequality problem in Euclidean space," Optimization, vol. 61, no. 9, pp. 1119-1132, 2012.

[12] Y. Yao, M. Postolache, and J. C. Yao, "Strong convergence of an extragradient algorithm for variational inequality and fixed point problems," University Politehnica of Bucharest Scientific Bulletin-Series A, vol. 82, no. 1, pp. 3-12, 2020.

[13] A. Gibali, S. Reich, and R. Zalas, "Iterative methods for solving variational inequalities in Euclidean space," Journal of Fixed Point Theory and Applications, vol. 17, no. 4, pp. 775-811, 2015.

[14] Q. L. Dong, Y. Peng, and Y. Yao, "Alternated inertial projection methods for the split equality problem," Journal of Nonlinear and Convex Analysis, vol. 22, pp. 53-67, 2021.

[15] X. Zhao, M. A. Köbis, Y. Yao, and J.-C. Yao, “A projected subgradient method for nondifferentiable quasiconvex multiobjective optimization problems," Journal of Optimization Theory and Applications, vol. 190, no. 1, pp. 82-107, 2021.

[16] D. Van Hieu, P. K. Anh, and L. D. Muu, "Modified hybrid projection methods for finding common solutions to variational inequality problems," Computational Optimization and Applications, vol. 66, no. 1, pp. 75-96, 2017.

[17] Y. Yao, J. C. Yao, Y.-C. Liou, and M. Postolache, "Iterative algorithms for split common fixed points of demicontractive operators without priori knowledge of operator norms," Carpathian Journal of Mathematics, vol. 34, no. 3, pp. 459466, 2018.

[18] A. N. Iusem and B. F. Svaiter, "A variant of Korpelevich's method for variational inequalities with a new search strategy," Optimization, vol. 42, no. 4, pp. 309-321, 1997.

[19] G. Fichera, "Sul problema elastostaticodi signorini conambigue condizionial contorno," Accounts of the Accademia Nazionale dei Lincei, Class of Physical, Mathematical and Natural Sciences, vol. 34, pp. 138-142, 1963.

[20] Y. Yao, M. Postolache, and J. C. Yao, "Iterative algorithms for the generalized variational inequalities," University Politehnica of Bucharest Scientific Bulletin-Series A, vol. 81, pp. 3-16, 2019.

[21] L.-C. Ceng, A. Petrusel, J. C. Yao, and Y. Yao, "Hybrid viscosity extragradient method for systems of variational inequalities, fixed points of nonexpansive mappings, zero points of accretive operators in Banach spaces," Fixed Point Theory, vol. 19, no. 2, pp. 487-502, 2018.

[22] T. M. M. Sow, "General viscosity methods for solving equilibrium problems, variational inequality problems and fixed point problems involving a finite family of multivalued strictly pseudo-contractive mappings," Journal of Advanced Mathematical Studies, vol. 13, pp. 275-293, 2020.

[23] L.-C. Ceng, A. Petrusel, J.-C. Yao, and Y. Yao, "Systems of variational inequalities with hierarchical variational inequality constraints for Lipschitzian pseudocontractions," Fixed Point Theory, vol. 20, no. 1, pp. 113-134, 2019.

[24] S. Y. Cho, X. Qin, J. C. Yao, and Y. Yao, "Viscosity approximation splitting methods for monotone and nonexpansive operators in Hilbert spaces," Journal of Nonlinear and Convex Analysis, vol. 19, pp. 251-264, 2018.

[25] E. N. Khobotov, "Modification of the extra-gradient method for solving variational inequalities and certain optimization problems," USSR Computational Mathematics and Mathematical Physics, vol. 27, no. 5, pp. 120-127, 1987.

[26] Y. Yao, M. Postolache, Y.-C. Liou, and Z. Yao, "Construction algorithms for a class of monotone variational inequalities," Optimization Letters, vol. 10, no. 7, pp. 1519-1528, 2016.

[27] P.-E. Maingé, "Numerical approach to monotone variational inequalities by a one-step projected reflected gradient method 
with line-search procedure," Computers \& Mathematics with Applications, vol. 72, no. 3, pp. 720-728, 2016.

[28] Y. Yao, M. Postolache, and J. C. Yao, "An iterative algorithm for solving the generalized variational inequalities and fixed points problems," Mathematics, vol. 7, p. 61, 2019.

[29] Y. Malitsky, "Projected reflected gradient methods for monotone variational inequalities," SIAM Journal on Optimization, vol. 25, no. 1, pp. 502-520, 2015.

[30] G. Stampacchia, "Forms bilineaires coercitives sur les ensembles convexes," Comptes Rendus de l'Academie des Sciences, Paris, vol. 258, pp. 4413-4416, 1964.

[31] D. V. Thong and D. V. Hieu, "New extragradient methods for solving variational inequality problems and fixed point problems," Journal of Fixed Point Theory and Applications, vol. 20, p. 129, 2018.

[32] Y. Yao, Y.-C. Liou, and J.-C. Yao, "Iterative algorithms for the split variational inequality and fixed point problems under nonlinear transformations," Journal of Nonlinear Sciences and Applications, vol. 10, no. 2, pp. 843-854, 2017.

[33] P. E. Maingé, "A hybrid extragradient-viscosity method for monotone operators and fixed point problems," SIAM Journal on Control and Optimization, vol. 47, pp. 1499-1515, 2008.

[34] X. Zhao, J. C. Yao, and Y. Yao, "A proximal algorithm for solving split monotone variational inclusions," University Politehnica of Bucharest Scientific Bulletin-Series A, vol. 82, no. 3, pp. 43-52, 2020.

[35] P. Tseng, "A modified forward-backward splitting method for maximal monotone mappings," SIAM Journal on Control and Optimization, vol. 38, no. 2, pp. 431-446, 2000.

[36] H. Zegeye, N. Shahzad, and Y. Yao, "Minimum-norm solution of variational inequality and fixed point problem in Banach spaces," Optimization, vol. 64, no. 2, pp. 453-471, 2015.

[37] C. Zhang, Z. Zhu, Y. Yao, and Q. Liu, "Homotopy method for solving mathematical programs with bounded box-constrained variational inequalities," Optimization, vol. 68, pp. 2293-2312, 2019.

[38] L. Zhang, C. Fang, and S. Chen, "An inertial subgradient-type method for solving single-valued variational inequalities and fixed point problems," Numerical Algorithms, vol. 79, no. 3, pp. 941-956, 2018.

[39] X. Zhao and Y. Yao, "Modified extragradient algorithms for solving monotone variational inequalities and fixed point problems," Optimization, vol. 69, pp. 1987-2002, 2020.

[40] M. V. Solodov and B. F. Svaiter, "A new projection method for variational inequality problems," SIAM Journal on Control and Optimization, vol. 37, no. 3, pp. 765-776, 1999.

[41] X. Cai, G. Gu, and B. He, "On the $O(1 / t)$ convergence rate of the projection and contraction methods for variational inequalities with Lipschitz continuous monotone operators," Computational Optimization and Applications, vol. 57, no. 2, pp. 339-363, 2014.

[42] G. M. Korpelevich, "The extragradient method for finding saddle points and other problems," Matecon, vol. 12, pp. 747-756, 1976.

[43] Y. Censor, A. Gibali, and S. Reich, "The subgradient extragradient method for solving variational inequalities in Hilbert space," Journal of Optimization Theory and Applications, vol. 148 , no. 2, pp. $318-335,2011$.

[44] Q.-L. Dong, D. Gibali, and D. Jiang, "A modified subgradient extragradient method for solving the variational inequality problem," Numerical Algorithms, vol. 79, no. 3, pp. 927-940, 2018.
[45] Y. Yao, X. Qin, and J. C. Yao, "Projection methods for firmly type nonexpansive operators," Journal of Nonlinear and Convex Analysis, vol. 19, pp. 407-415, 2018.

[46] P. E. Maingé and M. L. Gobinddass, "Convergence of one-step projected gradient methods for variational inequalities," Journal of Optimization Theory and Applications, vol. 171, no. 1, pp. 146-168, 2016. 